\title{
Molecular and ionized gas in the tidal tail in Stephan's Quintet
}

\author{
U. Lisenfeld ${ }^{1,2}$, J. Braine ${ }^{3}$, P.-A. Duc ${ }^{4}$, E. Brinks ${ }^{5}$, V. Charmandaris ${ }^{6,7}$, and S. Leon ${ }^{1}$ \\ ${ }^{1}$ Instituto de Astrofísica de Andalucía, CSIC, Apdo. 3004, 18040 Granada, Spain \\ 2 Dept. de Física Teórica y del Cosmos, Universidad de Granada, Granada, Spain \\ 3 Observatoire de Bordeaux, UMR 5804, CNRS/INSU, BP 89, 33270 Floirac, France \\ ${ }^{4}$ CNRS URA 2052 and CEA/DSM/DAPNIA Service d'Astrophysique, Saclay, 91191 Gif-sur-Yvette Cedex, France \\ 5 INAOE, Apdo. Postal 51 \& 216, Puebla, Pue 72000, Mexico \\ ${ }^{6}$ Cornell University, Astronomy Department, Ithaca, NY 14853, USA \\ 7 Chercheur Associé, Observatoire de Paris, LERMA, 61 Av. de l'Observatoire, 75014 Paris, France
}

Received 21 May 2004 / Accepted 7 July 2004

\begin{abstract}
We have mapped with the IRAM interferometer at Plateau de Bure (PdBI) the ${ }^{12} \mathrm{CO}$ emission towards intergalactic star forming regions located in the tidal tail stemming from NGC 7319, in the Stephan's Quintet compact group of galaxies. The ${ }^{13} \mathrm{CO}$ emission of the same region was observed with the IRAM $30 \mathrm{~m}$ telescope and optical spectroscopy of several HII regions in the area were obtained with the Calar Alto $3.5 \mathrm{~m}$ telescope. We recovered with the interferometer about $50 \%$ of the ${ }^{12} \mathrm{CO}(1-$ 0) total emission that had been earlier measured with single dish observations (Lisenfeld 2002, A\&A, 394, 823), indicating that about half of the molecular gas is distributed on spatial scales larger than about 10-15" (corresponding to 4-6 kpc) to which PdBI is not sensitive. We find two main areas of CO emission: (i) an elongated region towards the area known as SQ B where a Tidal Dwarf Galaxy could currently be forming and (ii) a barely resolved area at the tip of the optical tidal arm. Both regions follow dust lanes visible on HST images and their CO peak coincides spatially exactly with the maximum of the $\mathrm{H} \alpha$ line emission. In SQ B, there is furthermore very good kinematical agreement between the CO, $\mathrm{H} \alpha$ and $\mathrm{HI}$ components. We conclude from these coincidences that the gaseous matter found in quantities in the area is physically associated to the optical tidal tail and thus that the intergalactic atomic hydrogen there was expelled from NGC 7319. Its origin had previously been much debated. Furthermore, the relatively high oxygen abundances (about solar) estimated from the optical spectra of the HII regions imply that the gas feeding the star formation originated from the inner regions of the parent galaxy. In SQ B, we derive from different tracers a star formation rate, corrected for dust extinction - which is important in the area - of $0.5 M_{\odot} / \mathrm{yr}$, i.e. one of the highest values so far measured outside galaxies. The inferred molecular gas consumption time of $0.5 \mathrm{Gyr}$ lies in the range of values found for spiral and starburst galaxies. On the other hand, the ratio of ${ }^{12} \mathrm{CO} /{ }^{13} \mathrm{CO}>25$ is much higher than the values found in disks of spiral galaxies. A relatively low opacity for the ${ }^{12} \mathrm{CO}$ gas is the most likely reason.
\end{abstract}

Key words. stars: formation - ISM: molecules - galaxies: clusters: individual: Stephan's Quintet - galaxies: interactions galaxies: ISM - galaxies: intergalactic medium

\section{Introduction}

The Hickson Compact Group Stephan's Quintet (Hickson Compact Group 92; hereafter SQ) consists of four interacting galaxies (NGC 7319, NGC 7318a, NGC 7318b, and NGC 7317) and a foreground galaxy (NGC 7320). A fifth galaxy, NGC 7320c, situated $\sim 4$ arcmin to the east, is also dynamically associated to the group. The group has experienced a violent dynamical history with numerous interactions between the different members during the past Gyr (see Moles et al. 1997; Sulentic et al. 2001, for plausible scenarios). As a result of these interactions, two tidal arms, a faint older one, and a brighter young one stemming from NGC 7319 , have been created towards the eastern side of the group. Sulentic et al. (2001), suggest that each of these tails was created by a passage of NGC 7320c, the galaxy towards which both tails are pointing.
Several knots of star formation (SF) are visible in the young tidal arm extending from NGC 7319 to the east. The brightest region, identified as B by Xu et al. (1999) and hereafter called SQ B, shows also mid-infrared and $\mathrm{H} \alpha$ emission. This region has been identified as a good candidate for a Tidal Dwarf Galaxy (TDG) (Lisenfeld et al. 2002). Another fainter SF region is visible at the very tip of the tidal tail, hereafter called SQ tip.

One of the most striking properties of this group is that the major part of the gas is in the intragroup medium. Abundant atomic gas is present to the east of the three central galaxies. Williams et al. (2002) concluded from the HI kinematics that in reality this gas clouds consist of two subclouds, which they called Arc-N and Arc-S. Arc-N coincides at its southern end with the young tidal tail and Arc-S largely overlaps with the old tidal tail. In the northern part of Arc-N several HII regions are detected (Sulentic et al. 2001; Mendes de Oliveira et al. 2004). 
The origin of the gas is unclear. A plausible explanation for the gas in Arc-N is that it has been stripped from NGC 7319, the galaxy where the young tidal tail starts (Sulentic et al. 2001). A definite physical relation between Arc-N and the young stellar tail is however not proven, the apparent overlap could in principle be a projection effect. The only argument in favor of a real association is the fact that the sharp inner edge of the gas cloud follows the shape of the stellar optical tail (Sulentic et al. 2001). Part of the HI could also have been stripped from NGC 7320c, the other spiral galaxy in the group which completely lacks atomic gas.

Lisenfeld et al. (2002) observed the region around SQ B, close to the peak of the HI distribution, with the IRAM $30 \mathrm{~m}$ telescope and found abundant molecular gas $\left(7 \times 10^{8} M_{\odot}\right)$ covering a huge area of about $20 \mathrm{kpc}$. In this paper we present new millimeter observations of ${ }^{12} \mathrm{CO}$ with the IRAM interferometer at Plateau de Bure covering the region around SQ B and SQ tip. The goal of these observations was to map the distribution of the molecular gas at a higher spatial resolution and to study its relation to the SF regions in the tidal tails. Furthermore, we obtained optical spectroscopy of SQ B and SQ tip in order to further study the physics of the star forming regions, as well as observations of SQ B with the IRAM $30 \mathrm{~m}$ telescope of ${ }^{13} \mathrm{CO}$ in order to collect more information about the physical state of the molecular gas.

In Sect. 2, we describe the observations; in Sect. 3, we present the results; in Sect. 4, we discuss some of their implications and Sect. 5 gives a summary of our most important results and conclusions. We adopt a distance of $85 \mathrm{Mpc}$, based on a recession velocity of $6400 \mathrm{~km} \mathrm{~s}^{-1}$ to SQ and $H_{0}=$ $75 \mathrm{~km} \mathrm{~s}^{-1} \mathrm{Mpc}^{-1}$, in which case $10^{\prime \prime}$ correspond to $4.1 \mathrm{kpc}$.

\section{Observations and data reduction}

\subsection{Plateau de Bure Interferometer}

Observations of the eastern young tidal tail of SQ were carried out with the IRAM interferometer at Plateau de Bure (PdBI) ${ }^{1}$ between May and December 2002, using the CD set of configurations of the array.

We observed simultaneously the $J=1-0$ and $J=2-1$ lines of ${ }^{12} \mathrm{CO}$ in a single field centered at $\alpha_{\mathrm{J} 2000}=22^{\mathrm{h}} 36^{\mathrm{m}} 11.1^{\mathrm{s}}$ and $\delta_{\mathrm{J} 2000}=33^{\circ} 57^{\prime} 17^{\prime \prime}$. The primary beam size which gives the diameter beyond which the sensitivity of the instruments falls below half of its sensitivity at the center, is $44^{\prime \prime}\left(22^{\prime \prime}\right)$ in the 1-0 (2-1) line. The spectral correlator was split in two halves centered at 112.788 and $225.572 \mathrm{GHz}$, respectively, i.e., the transition rest frequencies corrected for an assumed recession velocity of $v_{\mathrm{o}}(\mathrm{LSR})=6600 \mathrm{~km} \mathrm{~s}^{-1}$. We observed each line in parallel with two correlator configurations: a high-resolution configuration with a frequency resolution of $0.312 \mathrm{MHz}$ and a bandwidth of $80 \mathrm{MHz}$, corresponding to a velocity resolution of $0.83 \mathrm{~km} \mathrm{~s}^{-1}$ and a bandwidth of $212 \mathrm{~km} \mathrm{~s}^{-1}$ at $\mathrm{CO}(1-0)$, and a low-resolution configuration with a frequency resolution of

\footnotetext{
${ }^{1}$ Based on observations carried out with the IRAM Plateau de Bure Interferometer. IRAM is supported by INSU/CNRS (France), MPG (Germany) and IGN (Spain).
}

$1.28 \mathrm{MHz}$ and a bandwidth of $320 \mathrm{MHz}$, corresponding to a velocity resolution of $3.32 \mathrm{~km} \mathrm{~s}^{-1}$ and a bandwidth of $830 \mathrm{~km} \mathrm{~s}^{-1}$ at $\mathrm{CO}(1-0)$. Since the high-resolution data were rather noisy, we only use the low-resolution data cube in this paper. Phase and amplitude calibrations were performed by observing the nearby quasars $2145+065,2201+315,2234+282$, 3C 454.3, 3C 273 and $1633+382$, as well as MWC 349. The fluxes of the primary calibrators were determined from IRAM $30 \mathrm{~m}$ measurements and taken as an input to derive the absolute flux density scale in our map; the latter is estimated to be accurate to $10 \%$. We estimate the uncertainty in the final flux calibration to be about $20 \%$.

The data of the ${ }^{12} \mathrm{CO}(2-1)$ line were of poor quality and we will not use them in the analysis presented in this paper. The image reconstruction was done using standard IRAM/Gildas software. We used natural weighting and no taper to generate the ${ }^{12} \mathrm{CO}(1-0)$ line maps with a $0.5^{\prime \prime}$ sampling. The corresponding synthesized beam is $4.33^{\prime \prime} \times 3.46^{\prime \prime}$, PA $=-85^{\circ}$. During the process of data reduction we have also tested uniform weighting and different taperings in order to search for weaker emission features, but the resulting maps were not significantly different. The data, except for the displayed maps, are corrected for primary beam attenuation.

\subsection{IRAM $30 \mathrm{~m}$ telescope}

We observed the ${ }^{13} \mathrm{CO}(1-0)$ and ${ }^{13} \mathrm{CO}(2-1)$ towards SQ B (centered at $\alpha_{\mathrm{J} 2000}=22^{\mathrm{h}} 36^{\mathrm{m}} 10.3^{\mathrm{s}}$ and $\left.\delta_{\mathrm{J} 2000}=33^{\circ} 57^{\prime} 17^{\prime \prime}\right)$ with the IRAM 30 m telescope on Pico Veleta in March 2004 under excellent weather conditions, with system temperatures of $115 \mathrm{~K}$ at $108 \mathrm{GHz}$ and $210 \mathrm{~K}$ at $216 \mathrm{GHz}$ on the $T_{\mathrm{A}}^{*}$ scale. Dual polarization receivers were used at both frequencies with the $256 \times 1 \mathrm{MHz}$ filterbanks on each receiver. The observations were made with a wobbling secondary in beam switch mode with a wobbler throw of $70^{\prime \prime}$ in azimuthal direction and a wobbling frequency of $0.5 \mathrm{~Hz}$. Pointing was checked every $60-90 \mathrm{~min}$ on the nearby quasars $2251+158$, and was very good, with rms offsets of less than $3^{\prime \prime}$ on average. At the end of the observations, the frequency tuning was checked by observing Orion. The IRAM forward efficiency, $F_{\text {eff }}$, was 0.95 and 0.91 at 115 and $230 \mathrm{GHz}$ and the beam efficiency, $B_{\text {eff }}$, was 0.75 and 0.54 , respectively. The half-power beam size was $22^{\prime \prime}$ at $110 \mathrm{GHz}$ and $11^{\prime \prime}$ at $215 \mathrm{GHz}$. The CO spectra and luminosities are presented on the main beam temperature scale $\left(T_{\mathrm{mb}}\right)$ which is defined as $T_{\mathrm{mb}}=\left(F_{\mathrm{eff}} / B_{\mathrm{eff}}\right) \times T_{\mathrm{A}}^{*}$. For the data reduction, the spectra were summed and a constant continuum level was subtracted.

\subsection{Optical observations}

The spectra of the HII regions towards SQ B were obtained in July 2003 with the MOSCA instrument installed on the Calar Alto $3.5 \mathrm{~m}$ telescope. Using the multi-slit capabilities of the spectrograph, we obtained about 70 spectra of intergalactic HII regions in the field of SQ. Each region was observed with a medium (R1000, $1.5 \AA /$ pixel) and a low (B500, $2.9 \AA /$ pixel) resolution grism. Special care was taken in positioning the slits 


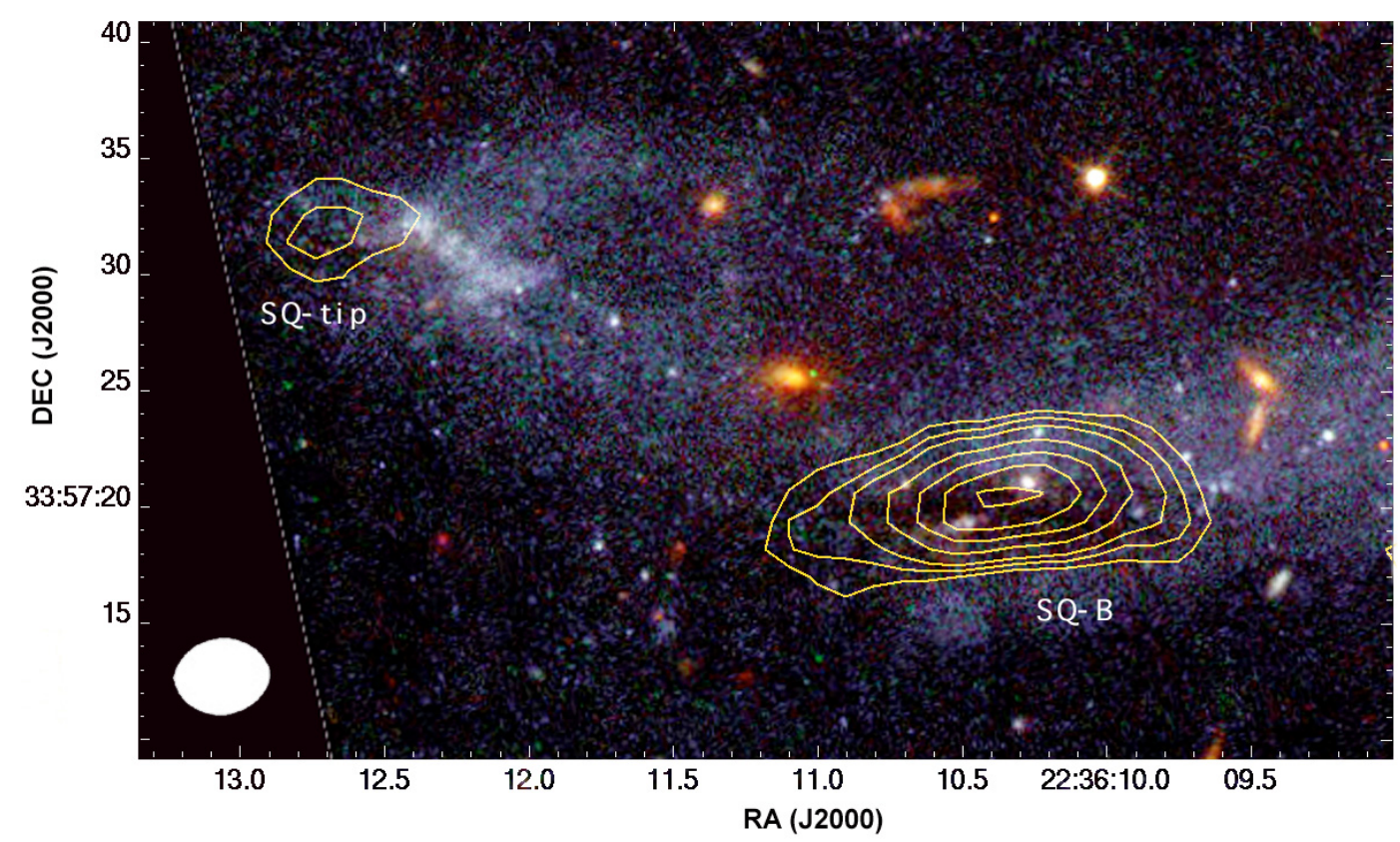

Fig. 1. Contours of the PdBI observations overlaid on a Hubble Space Telescope (HST) image (Gallagher et al. 2001). The velocity integration was carried out from 6570 to $6670 \mathrm{~km} \mathrm{~s}^{-1}$. The contour levels presented are at $2 \sigma, 3 \sigma, 4 \sigma$, and then increase in intervals of $2 \sigma$. Note that $2 \sigma\left(=0.18 \mathrm{Jy} \mathrm{km} \mathrm{s}^{-1}\right.$ beam $\left.^{-1}\right)$ corresponds to a surface density of $2 \times 10^{20} \mathrm{~cm}^{-2}$, and the maximum contour corresponds to $1.2 \times 10^{21} \mathrm{~cm}^{-2}$. The beam is shown in the lower left corner.

and extracting the spectra. Most HII regions have an extremely faint continuum, so that they could not be used to trace the spectrum along the dispersion direction. Hence, the aperture corrections were done by manually fitting the positions of the emission lines. The rest of the data reduction was carried out in a standard way using the IRAF software. Details of our spectroscopic study will be presented in a forthcoming paper (Duc et al., in preparation), and here we only report on results relevant to SQ B.

\section{Results}

\subsection{Distribution and mass of the molecular gas}

Figure 1 shows the velocity integrated intensity map of the PdBI observations overlaid over an optical Hubble Space Telescope (HST) image (Gallagher et al. 2001). The CO emission is distributed mainly in two regions. The most prominent emission region has an elongated shape that roughly follows the tidal tail with the maximum centered on a dust lane crossing SQ B. Its overall extent is $\sim 18^{\prime \prime} \times 7^{\prime \prime}$ or $\sim 7 \times 3 \mathrm{kpc}$. A much fainter emission region is visible at the tip of the tidal tail, SQ tip, slightly offset to the west from the tail and also coinciding with a dust lane. The integrated emission at this position is weak, but $\mathrm{CO}$ emission is clearly visible in the individual spectra. The molecular gas distribution with dimensions of about $5^{\prime \prime} \times 7^{\prime \prime}(2 \times 3 \mathrm{kpc})$ is only barely resolved by our beam. The channel maps (Fig. 2) reveal that the distribution of the $\mathrm{CO}$ within SQ B is not completely smooth but shows substructures appearing at different velocities.

In order to derive the total molecular gas masses in the different regions we have summed the spectra from the low velocity resolution data cube over the area where emission is visible in individual spectra. These summed spectra were then velocity integrated over the velocity range where emission was visible (6570 to $6670 \mathrm{~km} \mathrm{~s}^{-1}$ ) and the molecular mass was calculated assuming (as throughout this paper) the Galactic conversion factor of $N\left(\mathrm{H}_{2}\right) / I_{\mathrm{CO}}=2 \times 10^{20} \mathrm{~cm}^{-2}\left(\mathrm{~K} \mathrm{~km} \mathrm{~s}^{-1}\right)^{-1}$. The formula used to calculate the molecular gas mass from the velocity integrated flux, $S_{\mathrm{CO}}$, is:

$M_{\mathrm{H}_{2}}=7.8 \times 10^{3}(D / \mathrm{Mpc})^{2} S_{\mathrm{CO}} M_{\odot}$,

where $D$ is the distance in Mpc. This formula corresponds to the one derived in Braine et al. (2001, Eq. (4)) multiplied by a factor 0.73 in order to neglect the helium mass. The derived line fluxes and molecular gas masses are listed in Table 1.

The total molecular mass detected by the PdBI observations is $(3.3 \pm 0.7) \times 10^{8} M_{\odot}$. The total mass derived from the IRAM $30 \mathrm{~m}$ observations (Lisenfeld et al. 2002), is (7.0 \pm 1.4) $\times 10^{8} M_{\odot}$, showing that the PdBI observations pick up only about half of the total $\mathrm{CO}$ emission, the rest being distributed on scales larger than about $10-15^{\prime \prime}$ and therefore resolved out by the interferometer.

In order to better compare the spatial distribution of the compact and smoothly distributed emission in SQ B and SQ tip, we have co-added in each case the $\mathrm{CO}$ emission of the $30 \mathrm{~m}$ observations over 2 beams, covering the area where emission was detected by the PdBI in SQ B and SQ tip. Table 1 summarizes the molecular gas masses derived from the IRAM $30 \mathrm{~m}$ and $\mathrm{PdBI}$ in the individual regions. Due to the poor spatial resolution of the $30 \mathrm{~m}$ observations, the corresponding regions do not coincide completely (the $30 \mathrm{~m}$ data covers a larger area). The molecular gas masses observed at SQ B with PdBI is $65 \pm 22 \%$ of the mass detected with the $30 \mathrm{~m}$ telescope in a slightly larger area. At the tip of the tidal tail, the PdBI 


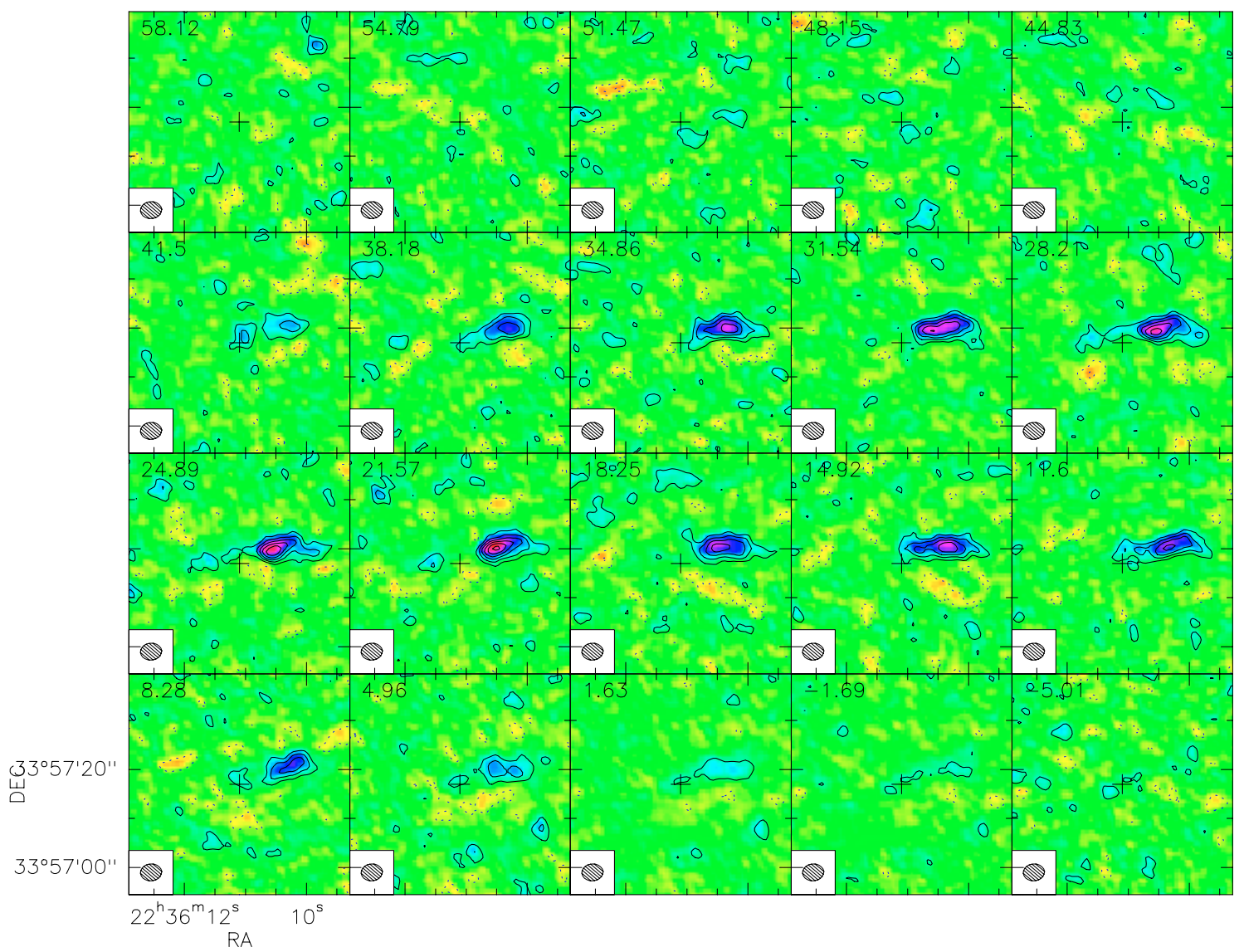

Fig. 2. Channel maps with a velocity resolution of $3.3 \mathrm{~km} \mathrm{~s}^{-1}$. The velocity is indicated at the upper left of each panel, given relative to $6600 \mathrm{~km} \mathrm{~s}^{-1}$. The beam shape is given in the lower left corner. The contour levels start at $6.7 \mathrm{mJy} / \mathrm{beam}(1 \sigma)$ and increase in intervals of $1 \sigma$. The cross indicates the center of the observations at $\alpha_{\mathrm{J} 2000}=22^{\mathrm{h}} 36^{\mathrm{m}} 11.1^{\mathrm{s}}$ and $\delta_{\mathrm{J} 2000}=33^{\circ} 57^{\prime} 17^{\prime \prime}$.

Table 1. Molecular gas masses in different regions.

\begin{tabular}{|c|c|c|c|}
\hline \multirow[t]{2}{*}{ Region } & \multicolumn{2}{|c|}{ PdBI } & IRAM $30 \mathrm{~m}$ \\
\hline & $\begin{array}{c}\text { Flux } \\
{\left[\mathrm{Jy} \mathrm{km} \mathrm{s}^{-1}\right]}\end{array}$ & $\begin{array}{c}M_{\mathrm{H}_{2}} \\
10^{8} M_{\odot}\end{array}$ & $\begin{array}{c}M_{\mathrm{H}_{2}} \\
10^{8} M_{\odot}\end{array}$ \\
\hline SQ B & $4.4 \pm 1.0$ & $2.5 \pm 0.6$ & $3.9 \pm 0.9$ \\
\hline SQ tip & $1.5 \pm 0.4$ & $0.8 \pm 0.2$ & $1.9 \pm 0.5$ \\
\hline Total & $5.9 \pm 1.3$ & $3.3 \pm 0.7$ & $7.0 \pm 1.4^{*}$ \\
\hline
\end{tabular}

The error includes both the formal error based on the rms noise in the integrated spectrum, the uncertainty in the calibration (20\%) and, for the PdBI data, an estimate for uncertainties due to different parameters in the data reduction, above all during the cleaning process $(10 \%)$.

(*) Refers to the central 15 beams observed with the $30 \mathrm{~m}$ telescope (see Lisenfeld et al. 2002).

observations detect a slightly lower fraction of about $40 \pm 14 \%$ of the total CO. Thus, within the uncertainties, the fraction of molecular gas picked up by the interferometer is not significantly different in both regions.

\section{2. ${ }^{13} \mathrm{CO}$ in $S Q B$}

The IRAM $30 \mathrm{~m}$ observations of ${ }^{13} \mathrm{CO}(2-1)$ resulted in a $3 \sigma$ detection. As shown in Fig. 3 the central line velocity agrees very well with the ${ }^{12} \mathrm{CO}(2-1)$ line observed at the same position in Lisenfeld et al. (2002), so we are confident that

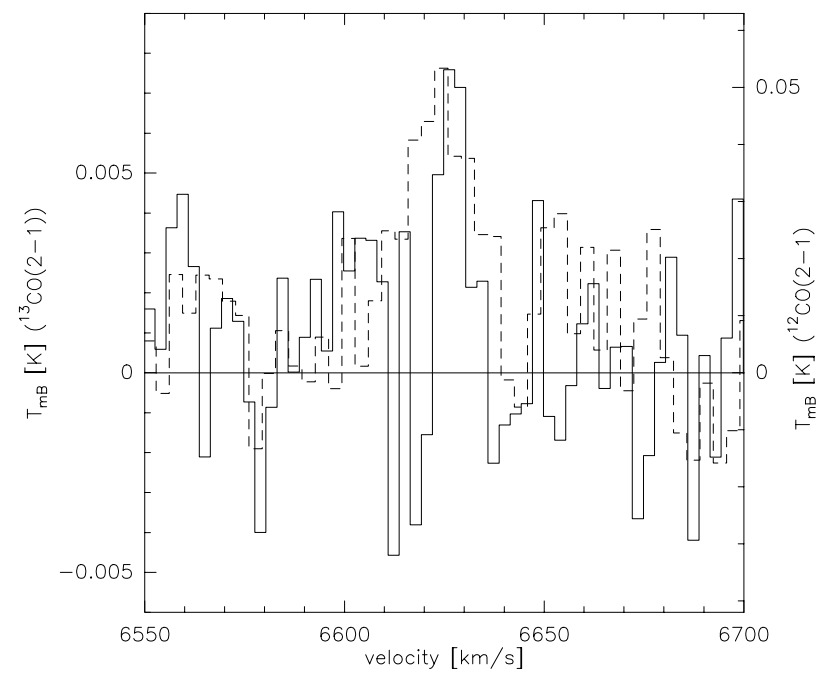

Fig. 3. The ${ }^{13} \mathrm{CO}(2-1)$ spectrum at SQ B (solid line, left temperature scale is applicable) compared to the spectrum of the ${ }^{12} \mathrm{CO}(2-1)$ line (dashed line, right temperature scale) at the same position. The velocity resolution in $2.78 \mathrm{~km} \mathrm{~s}^{-1}$.

the line is really detected. The velocity integrated intensity of ${ }^{13} \mathrm{CO}(2-1)$ is $0.06 \pm 0.02 \mathrm{~K} \mathrm{~km} \mathrm{~s}^{-1}$. However, no ${ }^{13} \mathrm{CO}(1-0)$ was detected, down to a rms noise level of $1 \mathrm{mK}$ at a velocity 
resolution of $10 \mathrm{~km} \mathrm{~s}^{-1}$, which corresponds to an upper limit of $0.04 \mathrm{~K} \mathrm{~km} \mathrm{~s}^{-1}$.

We can calculate the line ratios between the ${ }^{12} \mathrm{CO}$ and ${ }^{13} \mathrm{CO}$ emission from the velocity integrated intensities of the ${ }^{12} \mathrm{CO}$ lines at the same position: $I\left({ }^{12} \mathrm{CO}(1-0)\right)=1.1 \pm 0.1$ and $I\left({ }^{12} \mathrm{CO}(1-0)\right)=1.5 \pm 0.2$ (Lisenfeld et al. 2002). We find that ${ }^{12} \mathrm{CO}(1-0) /{ }^{13} \mathrm{CO}(1-0)>28$ and ${ }^{12} \mathrm{CO}(2-1) /{ }^{13} \mathrm{CO}(2-1)=25 \pm 9$. These values are much higher than the value of $\sim 10$ that is typically found in disks of spiral galaxies (e.g. Paglioni et al. 2001), and it is close to the values found for luminous infrared galaxies (Casoli et al. 1992).

\subsection{Properties of the ionized gas}

In SQ B, four individual HII condensations are clearly visible on the $\mathrm{H} \alpha$ narrow band image (see Fig. 4). We obtained an optical spectrum of each one of them. All spectra are characterized by a weak continuum and strong emission lines. The equivalent width of the $\mathrm{H} \alpha$ line exceeds $200 \AA$. Optical velocities (in the Local Standard of Rest, LSR, system) were derived from the redshifts of the Balmer lines in the medium-resolution spectra. They range between $6575-6765 \mathrm{~km} \mathrm{~s}^{-1}$. The brightest HII region has an average velocity of $6625 \mathrm{~km} \mathrm{~s}^{-1}$ (range: 6575-6655 $\mathrm{km} \mathrm{s}^{-1}$ ).

Assuming an intrinsic value of 2.85 for the Balmer decrement $\mathrm{H} \alpha / \mathrm{H} \beta$, in agreement with Case B recombination models (Osterbrock 1989), we derive an $A_{\mathrm{V}}$ as high as 3 mag for SQ B, consistent with the presence of a dust lane. The oxygen abundance of SQ B was determined empirically based on the $\left[\mathrm{N}_{\mathrm{II}}\right] / \mathrm{H} \alpha$ flux ratio and the calibration of van Zee et al. (1998). We obtained a value of $12+\log (\mathrm{O} / \mathrm{H})=8.7$ which is nearly solar (given the latest value of the oxygen abundance in the Sun, Allende Prieto et al. 2001). This value is rather high: HII regions along tidal tails have typically a $12+\log (\mathrm{O} / \mathrm{H})=8.4-8.6$ (Duc et al. 2004). It implies that the gas that made SQ B was not expelled from the outer regions of its parent galaxy, but rather from inside the disk.

\subsection{Comparison between the ionized and neutral gas}

In Fig. 4 we present a contour overlay of the $\mathrm{CO}$ emission, detected by the PdBI, to the $\mathrm{H} \alpha$ emission (Iglesias-Páramo \& Vílchez 2001, greyscale). Contours of the HI emission detected by Williams et al. (2002) are also shown with dashed lines. The exact spatial coincidence of the $\mathrm{CO}$ emission with the $\mathrm{H} \alpha$ emission in both SQ B and SQ tip is striking. Since dust is most likely coexisting with the molecular gas, this close correspondence is consistent with the high extinction obtained from the optical spectroscopy. The presence of dust is also clearly visible in SQ B in the optical image, where the peak of the $\mathrm{CO}$ emission coincides with a dust lane (see Fig. 1). We can estimate the expected extinction at the peak of the $\mathrm{CO}$ emission from the gas surface density adopting a Galactic extinction coefficient of $4.5 \times 10^{-22} \mathrm{mag} \mathrm{cm}^{2}$ in the $R$-band (Draine 2003). From the HI data we derive a column density of $7 \times 10^{20} \mathrm{~cm}^{-2}$ for SQ B and our PdBI CO data correspond to a peak of $17 \times 10^{20} \mathrm{~cm}^{-2}$ at the same region. Assuming a foreground

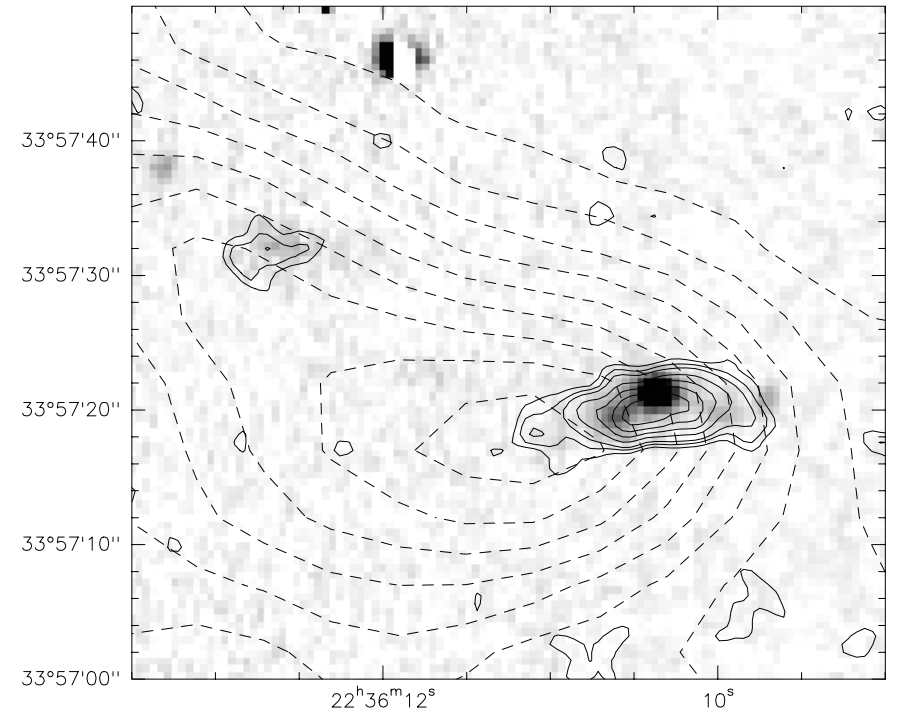

Fig. 4. Contours of the PdBI observations overlaid over an $\mathrm{H} \alpha$ image (from Iglesias-Páramo \& Vílchez 2001). The CO contours are as in Fig. 1. The dashed contours give the HI emission with levels starting at $1 \times 10^{20}$ atoms $\mathrm{cm}^{-2}$, and continuing in intervals of $1 \times 10^{20}$ until $9 \times 10^{20}$ atoms cm${ }^{-2}$.

screen of dust, this implies an extinction of $A_{\mathrm{V}}=2.3 \mathrm{mag}$, slightly lower but in the similar range as the value found from the Balmer decrement. In SQ tip the observed column densities of $N_{\mathrm{HI}}=6 \times 10^{20} \mathrm{~cm}^{-2}$ and $2 N_{\mathrm{H}_{2}}=8 \times 10^{20} \mathrm{~cm}^{-2}$ result in a somewhat lower extinction of $A_{\mathrm{V}}=1.3 \mathrm{mag}$.

The eastern end of the CO emission in SQ B coincides with the HI peak and extends towards the west in a region with a steep gradient in the HI distribution as already noted in Lisenfeld et al. (2002). This gradient goes along the optical tidal arm. Also in SQ tip, the CO emission lies at the end of this gradient.

\subsection{Gas kinematics}

In Figs. 5 and 6 we show the spatially integrated spectra of SQ B and SQ tip, observed with the PdBI and the IRAM $30 \mathrm{~m}$ telescope (solid and dashed lines respectively). In each case the IRAM $30 \mathrm{~m}$ spectra are the sum of the two beams that cover the area with $\mathrm{CO}$ emission detected by PdBI. In addition we show with a dotted line the total HI spectra, co-added over the same area.

Clearly in SQ B both CO spectra agree very well in shape, central velocity and line width. The same is true for the total emission in this region detected with the $30 \mathrm{~m}$ telescope (summing the emission of 15 beams that cover an area of about $60^{\prime \prime} \times 40^{\prime \prime}$, see Lisenfeld et al. 2002, Fig. 6) which extends over a larger region. The HI line has the same central velocity as the $\mathrm{CO}$ emission but it is slightly broader, which could be due to the poorer velocity resolution of this dataset. The central velocity and velocity range found for the molecular and atomic gas agree perfectly with the $\mathrm{H} \alpha$ emission of the strongest Hir region (see Table 2). The range of the other HII regions extends to somewhat higher velocities. 


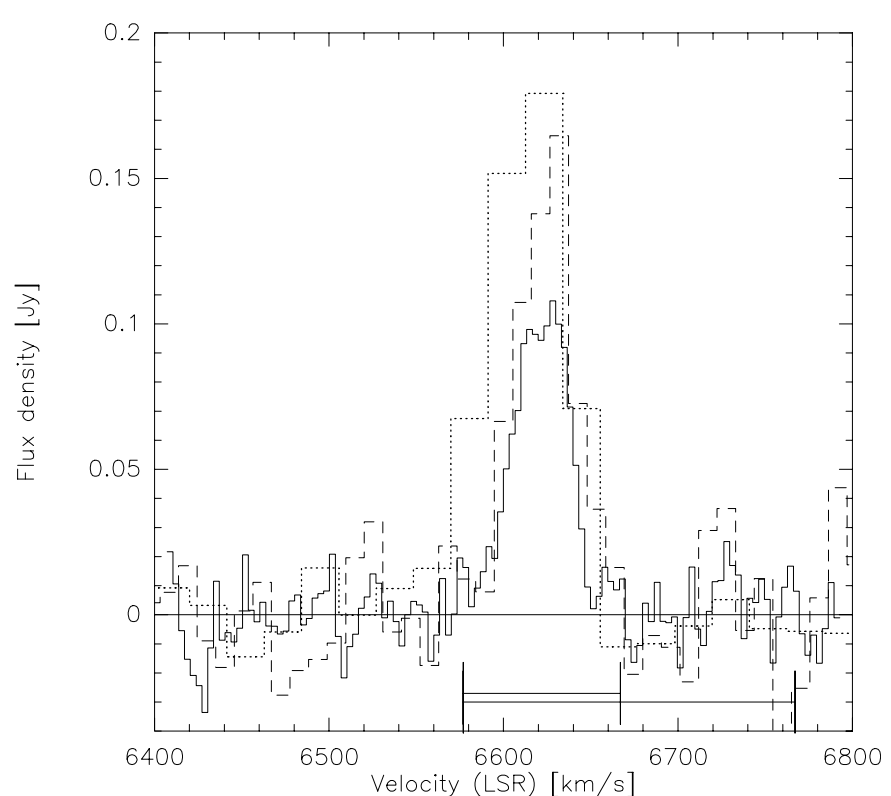

Fig. 5. The ${ }^{12} \mathrm{CO}(1-0)$ spectrum of PdBI with a velocity resolution of $3.3 \mathrm{~km} \mathrm{~s}^{-1}$ (solid line) integrated over the emission in SQ B, together with the $30 \mathrm{~m}$ spectrum of this region (dashed line) and the HI spectrum (dotted line) integrated over the same area as the $30 \mathrm{~m}$ data. The HI spectrum is scaled such that for equal flux density with the $30 \mathrm{~m}$ spectrum the corresponding $\mathrm{HI}$ surface density is twice the one of $\mathrm{H}_{2}$. The upper horizontal bar at the bottom indicates the velocity range of the $\mathrm{H} \alpha$ in the brightest $\mathrm{HII}$ regions, and the lower horizontal bar gives the velocity range of all HII regions in SQ B.

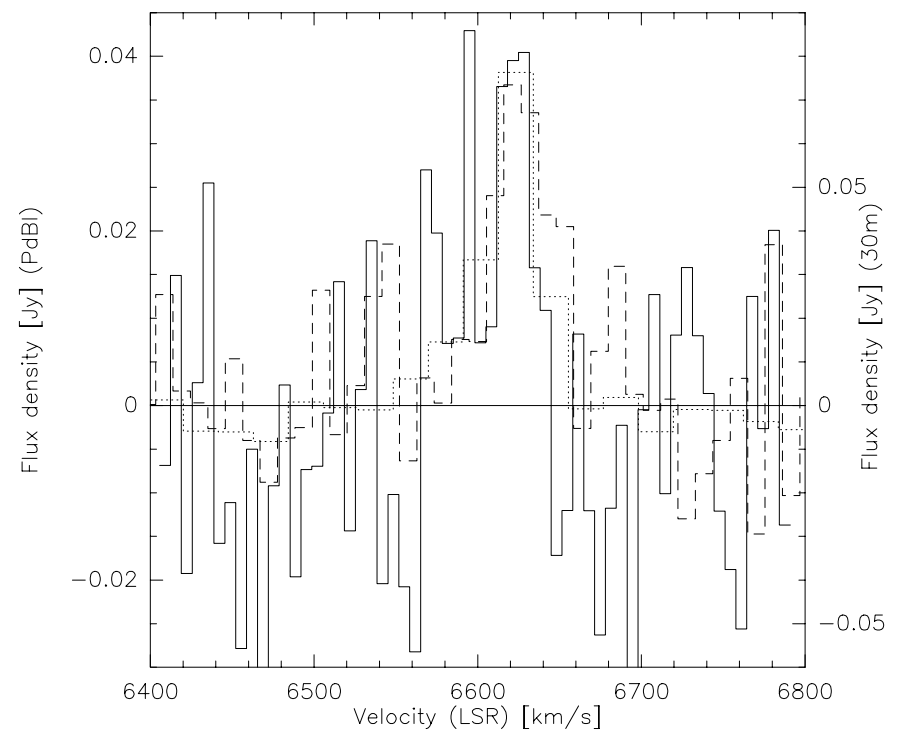

Fig. 6. CO spectrum of PdBI with a velocity resolution of $3.3 \mathrm{~km} \mathrm{~s}^{-1}$ (solid line) integrated over the emission in SQ tip, together with the $30 \mathrm{~m}$ spectrum over this region (dashed line) and the HI spectrum (dotted line) integrated over the same area as the $30 \mathrm{~m}$ data. The HI spectrum is scaled such that for equal flux density with the $30 \mathrm{~m}$ spectrum the corresponding $\mathrm{HI}$ surface density is 7 times the one of $\mathrm{H}_{2}$.

Even though the CO spectra of SQ tip are noisier (see Fig. 6), we also see that the central velocity and line shape are in very good agreement between the CO spectrum from $\mathrm{PdB}$, the $\mathrm{CO}$ spectrum from the $30 \mathrm{~m}$, and with the $\mathrm{HI}$ line.
Table 2. Kinematics of the molecular and ionized gas.

\begin{tabular}{ccccc}
\hline \hline & \multicolumn{2}{c}{ CO } & \multicolumn{2}{c}{$\mathrm{H} \alpha$} \\
& $\begin{array}{c}\text { Central } \\
\text { velocity } \\
{\left[\mathrm{km} \mathrm{s}^{-1}\right]}\end{array}$ & $\begin{array}{c}\text { Velocity } \\
\text { range } \\
{\left[\mathrm{km} \mathrm{s}^{-1}\right]}\end{array}$ & $\begin{array}{c}\text { Central } \\
\text { velocity } \\
{\left[\mathrm{km} \mathrm{s}^{-1}\right]}\end{array}$ & $\begin{array}{c}\text { Velocity } \\
\text { range } \\
{\left[\mathrm{km} \mathrm{s}^{-1}\right]}\end{array}$ \\
\hline SQ B & 6625 & $6575-6665$ & $6625^{1}$ & $6575-6655^{1}$ \\
& & & - & $6575-6765^{2}$ \\
SQ tip & 6625 & $6565-6640$ & - & - \\
\hline
\end{tabular}

(1) For the brightest HII region coinciding with the peak of CO.

(2) Refers to all 4 HII regions.

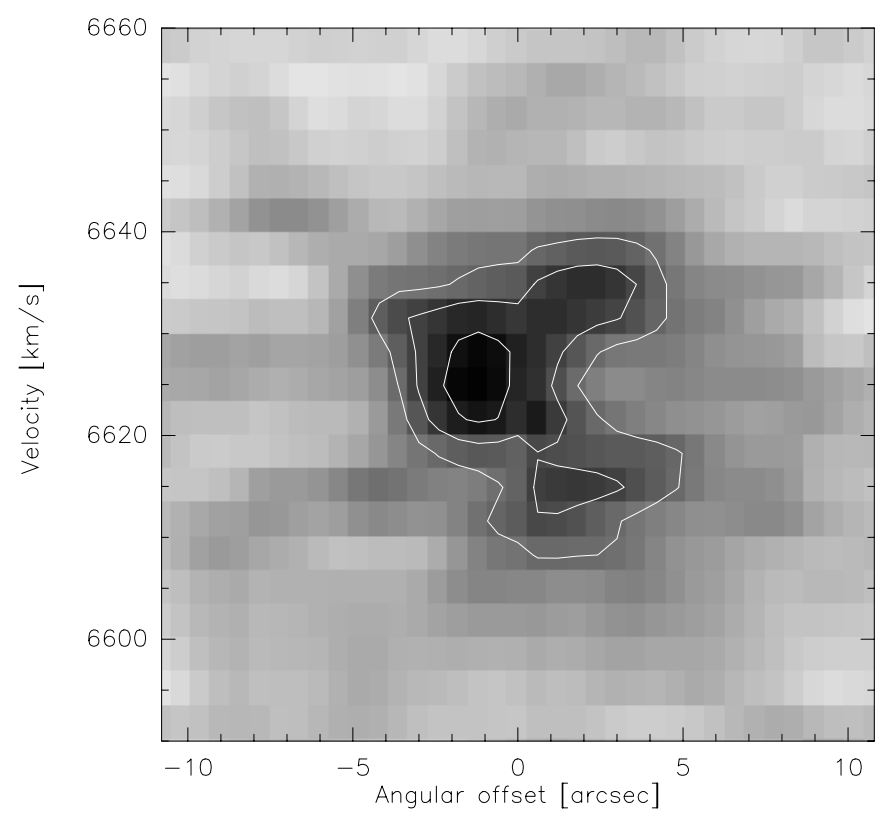

Fig. 7. Position-velocity diagram at SQ B along the east-west direction with a velocity resolution of $3.3 \mathrm{~km} \mathrm{~s}^{-1}$. The contour levels start at $20 \mathrm{mJy} /$ beam $(3 \sigma)$ and increase by $6.7 \mathrm{mJy} /$ beam $(1 \sigma)$.

We have inspected the CO data cube from PdBI and searched for velocity gradients by inspecting the channel maps and by producing various position-velocity (pv) diagrams at various position angles. In SQ tip the emission is too weak to study the kinematics in detail. In Fig. 7 we show the pvdiagram of SQ B obtained in the east-west direction. It shows an interesting V-shape feature, visible towards the western side as a gap in velocities in the range between $\sim 6615$ and $\sim 6630 \mathrm{~km} \mathrm{~s}^{-1}$. The interpretation of this feature is not clear. A possibility could be two components, one showing a velocity gradient and a second superposed component with a velocity offset of $25 \mathrm{~km} \mathrm{~s}^{-1}$. The $\mathrm{H} \alpha$ data also suggest a complex velocity structure, with large velocity gradients up to $200 \mathrm{~km} \mathrm{~s}^{-1}$ which need however to be confirmed by Fabry-Perot observation in order to exclude that they are artifacts.

\subsection{Star formation rate and efficiency}

The star formation rate (SFR) at the two regions we detected in $\mathrm{CO}$ can be estimated using a number of tracers. The $\mathrm{H} \alpha$ emission is most commonly used, even though it 
Table 3. Star formation rates derived from different tracers.

\begin{tabular}{lll}
\hline \hline Tracer & $\begin{array}{l}\text { SFR(SQ B) } \\
{\left[M_{\odot} / \mathrm{yr}\right]}\end{array}$ & $\begin{array}{l}\text { SFR(SQ tip) } \\
{\left[M_{\odot} / \mathrm{yr}\right]}\end{array}$ \\
\hline $\mathrm{H} \alpha^{1}$ & $9.6 \times 10^{-2}$ & $2.9 \times 10^{-2}$ \\
$\mathrm{H} \alpha^{2}$ & 0.5 & $7.3 \times 10^{-2}$ \\
$\mathrm{H} \alpha^{3}$ & 0.8 & - \\
$15 \mu \mathrm{m}$ & 0.5 & - \\
Radio continuum & 0.6 & - \\
\hline
\end{tabular}

\footnotetext{
${ }^{1}$ No extinction correction.

${ }^{2}$ Extinction correction of $A_{\mathrm{V}}=2.3 \mathrm{mag}$ (SQ B) and $A_{\mathrm{V}}=1.3 \mathrm{mag}$ (SQ tip), based on the gas surface density.

${ }^{3}$ Extinction correction of $A_{\mathrm{V}}=3$ mag derived from Balmer decrement.
}

suffers from extinction. The total $\mathrm{H} \alpha$ emission in SQ B and SQ tip is $1.2 \times 10^{40} \mathrm{erg} \mathrm{s}^{-1}$ and $3.6 \times 10^{39} \mathrm{erg} \mathrm{s}^{-1}$, respectively. The first value is from $\mathrm{Xu}$ et al. (1999) whose $\mathrm{H} \alpha$ image only covered SQ B but not SQ tip, and is corrected for NII contamination, assuming [NII6538]/H $\alpha=0.4$. The second one is from Iglesias-Páramo \& Vílchez (2001), after calibrating their deep $\mathrm{H} \alpha$ image using the SQ B flux derived by Xu et al. (1999). We can convert these $\mathrm{H} \alpha$ fluxes to a SFR, using the usual formula $S F R=3.0 \times 10^{-8}\left(L_{\mathrm{H} \alpha} / L_{\odot}\right) M_{\odot} \mathrm{yr}^{-1}$ of Kennicutt $(1998)$, which assumes an Initial Mass Function (IMF) with a Salpeter slope over the 0.1 to $100 M_{\odot}$ range. In Table 3 we present the SFR estimates for different extinction corrections.

In SQ B the SFR can also be measured using two extinction-free tracers, the thermal dust emission and the radio continuum. The luminosity at $15 \mu \mathrm{m}$ of SQ B is $L_{15} \mu \mathrm{m}=$ $8.2 \times 10^{8} L_{\odot}$ (Xu et al. 1999 , after correcting their value to our assumed distance). Applying the empirical relation of Roussel et al. (2001), $S F R=6.5 \times 10^{-9} L_{15 \mu \mathrm{m}} / L_{\odot}$, we obtained a value of $0.5 M_{\odot} \mathrm{yr}^{-1}$.

A final estimate for the SFR can be made from the radio continuum. Emission at $1.40 \mathrm{GHz}$ was detected from SQ B at a level of $0.6 \pm 0.2 \mathrm{mJy}(\mathrm{Xu}$ et al. 2003) corresponding to a radio luminosity of $5.2 \times 10^{20} \mathrm{~W} \mathrm{~Hz}^{-1}$. The spectral index between $1.40 \mathrm{GHz}$ and $4.86 \mathrm{GHz}$ of $0.7 \pm 0.4$ found by $\mathrm{Xu}$ et al. (2003) is typical for spiral galaxies (Condon 1992). We assume therefore that, as on average found for spiral galaxies, synchrotron radiation is responsible for $90 \%$ of the emission at $1.4 \mathrm{GHz}$ (Condon 1992). With this assumption, we can apply Eq. (18) of Condon (1992) to derive a $S F R$ of $0.6 M_{\odot} \mathrm{yr}^{-1}$, assuming a Salpeter IMF.

The SFRs in SQ B derived from the extinction-free tracers (dust emission and radio continuum) agree surprisingly well and indicate levels of SF in SQ B of about $0.5 M_{\odot} \mathrm{yr}^{-1}$. The same values are derived from the $\mathrm{H} \alpha$ after applying the extinction correction derived from the gas surface density, suggesting that this value is correct. This SFR is very high for an extragalactic object, much higher than the values observed in other TDGs, most of them of comparable or larger dynamical mass (Braine et al. 2001).

Based on the above values of the SFR we can derive the gas consumption time, defined as the molecular gas mass divided by the $S F R$ (i.e. the inverse of the star formation efficiency). In
SQ B, the (extinction-free) $S F R$ of $0.5 M_{\odot} \mathrm{yr}^{-1}$ and the molecular gas mass of $2.5 \times 10^{8} M_{\odot}$ (from the PdBI observations), yield a gas consumption time of 0.5 Gyr. In SQ tip, applying the extinction derived from the gas column density results to a $S F R$ of $0.073 M_{\odot} \mathrm{yr}^{-1}$ and with $M_{\mathrm{H}_{2}}=8 \times 10^{7} M_{\odot}$ the corresponding gas consumption time is $1.1 \mathrm{Gyr}$. This estimate though is rather uncertain given our limited knowledge of the extinction of the $\mathrm{H} \alpha$ flux and of the molecular gas conversion factor. Our measurment lies within the range of gas consumption times found for spiral galaxies, the average being 0.6 Gyr (adjusted to our value of the conversion factor) with a dispersion of a factor 3 (Kennicutt 1998). This result is still valid even if, as discussed in Sect. 4.2, our conversion factor overestimates the molecular gas content in SQ B. However, in this case the gas consumption time would be more similar to the values of $0.25 \mathrm{Gyr}$ with a dispersion of a factor of 2.5 derived for starburst galaxies by Kennicutt (1998). It is unlikely that the conversion factor is higher than the Galactic value due to the solar metallicity observed in SQ B.

\section{Discussion}

\subsection{Association with the optical tidal tail of NGC 7319}

On the basis of our observations we may be able to answer the question: is the north-eastern HI cloud (called Arc-N in Williams et al. 2002) physically associated with the optical tidal tail or not? This HI cloud overlaps at its southern end with the optical tidal tail, but it has been unclear whether this is simply a projection effect. The enormous size of this cloud makes it distinctly different from what is observed in tidal tails in other interacting systems. So far, the only argument in favor of a physical relation is the sharp inner edge of the gas cloud coinciding in position and shape with the stellar optical tail (Sulentic et al. 2001).

Our CO observations have shown that there is very good spatial and kinematical agreement between the $\mathrm{CO}$ and $\mathrm{H} \alpha$ at both SQ B and SQ tip. Furthermore, the line velocities and shapes of $\mathrm{CO}$ and $\mathrm{HI}$ match very well. From these results we conclude that the recent SF traced by the $\mathrm{H} \alpha$ is indeed fed by the molecular gas observed in $\mathrm{CO}$ and both are physically related to the atomic gas. Strictly speaking, this does not prove that these components are related to the older stars seen in the optical tidal arm. In principle, the optical tail could be a background object to the atomic, molecular and ionized gas, including the recent SF that it contains.

The main argument against such a projection effect is the fact that the spatial coincidence between the blue stellar knots in the optical tail at SQ B and SQ tip and the $\mathrm{H} \alpha$ emission occurs at two positions in the tidal tail. It is unlikely that this is due to a coincidence and we conclude that the $\mathrm{H} \alpha$ emission most likely has its origin within the optical tail. Since our observations establish a physical relation between the neutral and ionized gas, also the HI and molecular gas must therefore be related to the optical tail.

These results allow us to draw a further conclusion. The optical tidal tail stems from NGC 7319, which points at this galaxy as its progenitor. Since the gas and the optical tail are 
related, their origin is expected to be the same which suggests that the gas also comes from NGC 7319. The solar metallicity observed in SQ B furthermore indicates that the gas comes from relatively inner regions of this galaxy since an enrichment by recent SF is not sufficient to explain these values (see Braine et al. 2001).

\subsection{Physical conditions of the molecular gas}

The high ${ }^{12} \mathrm{CO} /{ }^{13} \mathrm{CO}$ line ratio derived both for the $(1-0)$ and the (2-1) line is similar to the values found in starburst galaxies (Aalto et al. 1995), including their extreme forms of infrared luminous (LIRGs) and ultraluminous (ULIRGs) galaxies (Solomon et al. 1997). In starburst and (U)LIRGs the ${ }^{12} \mathrm{CO}$ emission is believed to originate not from dense, selfgravitating molecular clouds, but instead from a moderately dense inter-cloud medium with a relatively low opacity (Aalto et al. 1995; Solomon et al. 1997). Dense molecular gas also exists in great abundance as shown by the strength of the line emission of dense gas tracers such as HCN (Gao \& Solomon 2004). Thus, these observations point to a two component interstellar medium (ISM): dense clouds where molecules as $\mathrm{HCN}$ emit and a diffuse intercloud medium which is, in contrast to normal galaxies, dense enough for the gas to be molecular. As a result of this moderate density, the molecular gas masses derived from the $\mathrm{CO}$ emission using a Galactic conversion factor are a factor of 3-5 too high (Braine \& Dumke 1998; Downes \& Solomon 1998).

An alternative explanation for the high ${ }^{12} \mathrm{CO} /{ }^{13} \mathrm{CO}$ line ratio is a different abundance of the ${ }^{13} \mathrm{C}$ isotope in starbursting galaxies (Casoli et al. 1992). These authors have proposed two possible processes that might be responsible for the underabundance of ${ }^{13} \mathrm{C}$ : (i) the selective production of ${ }^{12} \mathrm{C}$ in the young stars formed during the starburst; and (ii) the inflow of lowmetallicity gas with a high ${ }^{12} \mathrm{C} /{ }^{13} \mathrm{C}$ ratio from the outer regions into the central regions where the starburst takes place. In SQ B however, we know that the gas fueling the SF has a high metallicity and does therefore not originate from the outer regions of the parent galaxy so that process (ii) cannot be at work here. The increase in the ${ }^{12} \mathrm{C} /{ }^{13} \mathrm{C}$ abundance ratio due to process (i) depends very much on the adopted parameters (see the arguments in Sect. 5.2 of Casoli et al. 1992). Adopting a realistic value for the gas mass fraction converted into stars (20\%) and assuming that the IMF is not biased towards massive stars, we derive an increase in ${ }^{12} \mathrm{C} /{ }^{13} \mathrm{C}$ of not more than $10 \%$. Only in the case of an IMF strongly biased towards massive stars could this process account for the observed high ${ }^{12} \mathrm{CO} /{ }^{13} \mathrm{CO}$ line ratio in SQ B.

In the case of SQ B it seems therefore that a low density/opacity of the ISM is the most likely explanation for the low ${ }^{13} \mathrm{CO} /{ }^{12} \mathrm{CO}$ line ratio. As a consequence it is possible that the Galactic conversion overestimates the molecular gas masses. A comparison between SQ B and starburst galaxies is not unreasonable, since the SF activity in SQ B is indeed high, as indicated by the high equivalent width of the $\mathrm{H} \alpha$ line of $>200 \AA$. More line observations, in particular high-density tracers as $\mathrm{HCN}$, will be necessary to better understand the properties of the molecular gas in SQ B.
Interestingly, a high ${ }^{12} \mathrm{CO} /{ }^{13} \mathrm{CO}$ has also been found in a very different type of object, in the bridge of the interacting system Taffy galaxies (Braine et al. 2003). Here, the CO/HCN ratio is very low, in contrast to starburst and (U)LIRGs. At the same time only very little SF is found.

\section{Summary and conclusions}

We have presented our analysis of new high resolution $\left(4.3^{\prime \prime} \times\right.$ $\left.3.5^{\prime \prime}\right) \mathrm{CO}$ observations of the eastern tidal tail in SQ obtained with the interferometer at Plateau de Bure (PdBI), as well as optical spectroscopy of the two star forming (SF) regions in the zone, SQ B and SQ tip, along with observations of the ${ }^{13} \mathrm{CO}$ line with the IRAM $30 \mathrm{~m}$ telescope. The main results derived from our observations are:

(i) We have identified two main $\mathrm{CO}$ emitting regions, associated with regions of SF in the optical tail at SQ B and at the tip of the tidal tail, SQ tip. Our PdBI maps trace about half of the total $\mathrm{CO}$ emission derived from single dish 30 m IRAM observations (Lisenfeld et al. 2002). This indicates that about half of the molecular gas is distributed on scales larger than 10-15" for which PdBI is not sensitive. The spatial extent of this latter component is very large, covering an area of about $16 \times 25 \mathrm{kpc}$ (see Lisenfeld et al. 2002).

(ii) In both regions the $\mathrm{CO}$ coincides exactly with $\mathrm{H} \alpha$ emission. In SQ B there is furthermore perfect kinematical agreement with the $\mathrm{H} \alpha$ line. In addition, the line shapes and velocities of $\mathrm{CO}$ and of HI spectra (Williams et al. 2002) are identical. These agreements suggest that the recent SF and molecular and neutral gas are related. $\mathrm{H} \alpha$ and $\mathrm{CO}$ emission coincide with the blue star clusters visible in the optical tidal tail. Since this coincidence occurs at two different positions, it seems unlikely that it is a projection and we conclude that a physical relation to the optical tail exists. This relation suggests that the origin of the gas is NGC 7319, the galaxy from where the optical tail stems.

(iii) We tentatively detected ${ }^{13} \mathrm{CO}(2-1)$ in SQ B and obtained an upper limit for ${ }^{13} \mathrm{CO}(1-0)$. The ${ }^{13} \mathrm{CO}$ observations yield high values for the ${ }^{12} \mathrm{CO} /{ }^{13} \mathrm{CO}$ ratio of $\geq 25$, both for the 1-0 and for the 2-1 line, higher than those found in disks of spiral galaxies and comparable to those found in starburst galaxies. A low opacity of the ${ }^{12} \mathrm{CO}$ emitting gas is the most plausible explanation for this result, however more molecular line observations are necessary in order to draw firm conclusions.

(iv) Optical spectroscopy in SQ B and SQ tip revealed several knots of $\mathrm{H} \alpha$ emission. Unlike other TDGs, the metallicity of SQ B is roughly solar and the extinction derived from the optical spectra is high $\left(A_{V}=3.0\right)$. This indicates that the gas at SQ $B$ has its origin in relatively inner regions of the disk of the parent galaxy.

(v) From the extinction-corrected $\mathrm{H} \alpha$ emission and from extinction free SF tracers (dust emission and radio continuum) we derive a SFR of SQ B as high as $0.5 M_{\odot} / \mathrm{yr}$. The resulting gas consumption time of $0.5 \mathrm{Gyr}$ is in the range found for spiral and starburst galaxies by Kennicutt (1998). 
Acknowledgements. We would like to thank J. Iglesias-Páramo, J. Vílchez and C. K. Xu for making available their $\mathrm{H} \alpha$ data to us, L. Verdes-Montenegro for the HI data, J. Sulentic for helpful discussions, and the referee, Alessandro Boselli, for useful comments on the manuscript. U.L. and S.L. are supported by the Spanish MCyT Grant AYA 2002-03338 and by the Junta de Andalucía. V.C. would like to acknowledge the partial support of NASA through contract number 1257184 issued by JPL/Caltech and E.B. acknowledges financial support from CONACyT via project 27607-E.

\section{References}

Aalto, S., Booth, R. S., Black, J. H., \& Johansson, L. B. 1995, A\&A, 300, 369

Allende Prieto, C., Lambert, D. L., \& Asplund, M. 2001, ApJ, 556, L63

Braine, J., \& Dumke, M. 1998, A\&A, 333, 38

Braine, J., Duc, P.-A., Lisenfeld, U., et al. 2001, A\&A, 378, 51

Braine, J., Davoust, E., Zhu, M., et al. 2003, A\&A, 408, L13

Condon, J. J. 1992, ARA\&A, 30, 575

Casoli, F., Dupraz, C., \& Combes, F. 1992, A\&A, 264, 55

Downes, D., \& Solomon, P. M. 1998, ApJ, 507, 615

Draine, B. T. 2003, ARA\&A, 41, 241

Duc, P.-A., Bournaud, F., \& Masset, F. 2004, in Recycling intergalactic and interstellar matter, ed. P.-A. Duc, J. B., \& Brinks, E. (ASP), IAU Symp., 217, in press [arXiv: astro-ph/0402252]
Gallagher, S. C., Charlton, J. S., Hunsberger, S. D., Zaritsky, D., \& Withmore, B. C. 2001, AJ, 122, 163

Gao, Y., \& Solomon, P. M. 2004, ApJ, 606, 271

Iglesias-Páramo, J., \& Vílchez, P. 2001, ApJ, 550, 204

Kennicutt, R. C. 1998, ApJ, 498, 541

Lisenfeld, U., Braine, J., Duc, P. A., et al. 2002, A\&A, 394, 823

Mendes de Oliveira, C., Cypriano, E. S., Sodré, L. Jr., \& Balkowski, C. 2004, ApJ, 605, L17

Moles, M., Sulentic. J. W., \& Márquez, I. 1997, ApJ, 485, L69

Osterbrock, D. E. 1989, Astrophysics of gaseous nebulae and active galactic nuclei (Oxford: Oxford University Press)

Paglioni, T. A. D., Wall, W. F., Young, J. S., et al. 2001, ApJS, 135, 183

Rohlfs, K., \& Wilson, T. L., Tools of Radioastronomy (Heidelberg: Springer-Verlag)

Roussel, H., Sauvage, M., Vigroux, L., \& Bosma, A. 2001, A\&A, 372, 427

Solomon, P. M., Downes, D., Radford, S. J. E., \& Barrett, J. W. 1997, ApJ, 478, 144

Sulentic, J. W., Rosado, M., Dultzin-Hacyan, D., et al. 2001, AJ, 122, 2993

van Zee, L., Salzer, J. J., Haynes, M. P., O’Donoghue, A. A., \& Balonek, T. J. 1998, AJ, 116, 2805

Williams, B. A., Yun, M. S., Verdes-Montenegro, L., \& van Gorkom, J. H. 2002, AJ, 123, 2417

Xu, C., Sulentic, J.W., \& Tuffs, R. 1999, ApJ, 512, 178

Xu, C., Condon, J. J., Dopita, M., \& Tuffs, R. 2003, ApJ, 595, 665 